\title{
COMPARING PLEURAL FLUID CULTURE YIELD IN BEDSIDE INOCULATION AND STANDARD LABORATORY CULTURE
}

\author{
Hariharan Iyer1, Vijay Khatri2, Amita Athavale 3
}

${ }^{1}$ Senior Resident, Department of Chest Medicine and EPRC, Seth GS Medical College and KEM Hospital.

${ }^{2}$ Associate Professor, Department of Chest Medicine and EPRC, Seth GS Medical College and KEM Hospital.

3 Professor and HOD, Department of Chest Medicine and EPRC, Seth GS Medical College and KEM Hospital.

\begin{abstract}
\section{BACKGROUND}

Identification of the infecting bacteria by pleural fluid culture is very important for proper clinical care. But this is often unsuccessful, as conventional Gram stain and culture are negative in many cases. If there is an increase in the yield of culture by inoculating the pleural fluid into enrichment media, the spectrum of various organisms causing pleural infection and their antibiotic sensitivity can be identified and thus help in appropriate treatment.
\end{abstract}

\section{AIMS}

To compare the bacterial recovery rates from pleural fluids between bed side inoculation into enrichment media and into conventional processing.

\section{MATERIALS AND METHODS}

After a written informed consent a prospective study was carried out on 50 adult patients with pleural effusion attending a tertiary care centre. From aseptically tapped and collected pleural fluid at bed side, $5 \mathrm{ml}$ each was inoculated anaerobic media (ROBERTSONS cooked meat) a blood culture media. Additionally $5 \mathrm{ml}$ of pleural fluid was transported for further processing as per current practice i.e. in a sterile container. Growth in any of these media, was subjected to biochemical tests for species identification.

\section{RESULTS AND CONCLUSIONS}

Upon inoculation into sterile specimen container there were 5 cases (10\%) in which a growth was detected. 45 cases upon inoculation into sterile specimen container revealed no growth (90\%). Upon inoculation into Blood culture broth there were 6 cases (12\%) in whom a growth was detected. Thus by inoculating into Blood culture broth a two percent (2\%) increase in the yield was obtained. Upon inoculation into the Robertson's Cooked Meat media, there were two organisms which were isolated which were Non-Fermenter Gram negative bacilli. Thus it amounted to 4\% cases. These were not detected upon inoculation into sterile specimen container. All the isolated organisms were Gram Negative organisms in both conventional as well as enrichment media inoculation. Thus pleural fluid inoculation into enrichment media helps to identify organisms, which may not be detected on routine inoculation into sterile specimen container.

\section{KEYWORDS}

Pleural Fluid, Culture, Blood Culture Media, Robertson's Cooked Meat Media.

HOW TO CITE THIS ARTICLE: Iyer H, Khatri V, Athavale A. Comparing pleural fluid culture yield in bedside inoculation and standard laboratory culture. J. Evolution Med. Dent. Sci. 2016;5(79):5898-5901, DOI: 10.14260/jemds/2016/1331

\section{INTRODUCTION}

As many as $40 \%{ }^{(1)}$ of patients with bacterial pneumonia have an accompanying pleural effusion, more than $40 \%$ of patients with bacterial pneumonia and $60 \%$ of patients with pneumococcal pneumonia develop parapneumonic effusions.(2)

Bacterial spectrum of culture-positive parapneumonic pleural effusions have changed over time. Prior to the antibiotic era, Streptococcus pneumoniae was the most common. S pneumoniae and Staphylococcus aureus account

Financial or Other, Competing Interest: None.

Submission 26-08-2016, Peer Review 18-09-2016,

Acceptance 26-09-2016, Published 03-10-2016.

Corresponding Author:

Hariharan Iyer,

Department of Chest Medicine and EPRC,

Seth GS Medical College and KEM Hospital,

CVTC Building, Room No. 19, Ground Floor,

Parel East, Mumbai-12.

E-mail: hriyer25@gmail.com

DOI: $10.14260 /$ jemds $/ 2016 / 1331$

(c) (i) $($ ) for many of the aerobic gram-positive cultures. Presently, aerobic organisms are isolated slightly more frequently than anaerobic organisms. Klebsiella, Pseudomonas and Haemophilus species are the 3 most commonly isolated aerobic gram-negative organisms. Bacteroides and Peptostreptococcus species are the two most commonly isolated anaerobic organisms. $(3,4,5)$ The mortality and morbidity rates in patients with pneumonia and pleural effusion are higher than in patients with pneumonia alone.

The presence of microorganisms in the pleura is potentially life-threatening. Specimens such as pleural fluid often have low numbers of microorganisms and require processing in order to concentrate the microorganisms in the specimen.(6) Initiation of antimicrobial treatment may produce false negative results. $(7,8)$ Some organisms may be missed if proper media are not available.

Hence, it is important to know organisms infecting pleural fluid. Therefore, the methodology of finding the organisms has to be accurate. If there is an increase in the yield of culture by inoculating the pleural fluid into enrichment media, the spectrum of the various organisms 
causing pleural infection and their antibiotic sensitivity can be identified and thus help in appropriate treatment.

\section{AIMS AND OBJECTIVES}

To compare the bacterial recovery rates from pleural fluids between bedside inoculation into enrichment media and into conventional processing.

\section{MATERIALS AND METHODS}

After obtaining written informed consent a prospective study was carried out on 50 adult patients with pleural effusion attending a tertiary care centre. From aseptically tapped and collected pleural fluid at bedside, $5 \mathrm{~mL}$ each was inoculated into anaerobic media (Robertson's cooked meat) a blood culture media. Additionally, $5 \mathrm{~mL}$ of pleural fluid was transported for further processing as per current practice, i.e. in a sterile container.

In case of growth in any of these media, it was subjected to biochemical tests for species identification.

\section{Inclusion Criteria}

1. Clinical presentation compatible with pleural infection.

2. Patients above the age of 18 years.

\section{Exclusion Criteria}

1. Patient already on antibiotics at the time of tapping.

Patients were first subjected to a clinical history which included details of their chief complaints, past history of any significant respiratory illnesses, a general as well as systemic examination. Then a baseline CXR was done. Patients were then subjected to pleural tapping and the fluid was collected into routine sterile container and was simultaneously inoculated into enrichment media, i.e. Blood culture broth and RCM media and was submitted to microbiology for determining the growth if any in each of the media.

\section{ETHICS}

The study was approved by the Institutional Ethics Committee (IEC) 1 of the Seth GS Medical College and KEM Hospital, Mumbai. Approval letter dated IEC (I)/OUT/660/13. Project no. EC/63/13.

\section{RESULTS}

\begin{tabular}{|c|c|c|}
\hline Gender & Frequency & Percent \\
\hline Female & 14 & 28 \\
\hline Male & 36 & 72 \\
\hline Total & 50 & 100 \\
\hline \multicolumn{3}{|c|}{ Table 1: Gender Wise Distribution of Subjects } \\
\hline
\end{tabular}

\begin{tabular}{|c|c|c|}
\hline Age Group & Frequency & Percent \\
\hline Less than 20 years & 2 & 4 \\
\hline 20-30 years & 19 & 38 \\
\hline 30-40 years & 16 & 3 \\
\hline 40-50 years & 8 & 16 \\
\hline 50-60 years & 4 & 8 \\
\hline More than 60 years & 1 & 2 \\
\hline Total & $\mathbf{5 0}$ & $\mathbf{1 0 0}$ \\
\hline \multicolumn{2}{|c}{ Table 2: Age Wise Distribution of Subjects } \\
\hline
\end{tabular}

Analysis of the various organisms grown in different media and their comparison.

\begin{tabular}{|c|c|c|}
\hline Organisms & Frequency & Percentage \\
\hline E. coli & 1 & 2 \\
\hline E. coli + Pseudomonas & 1 & 2 \\
\hline Pseudomonas & 3 & 6 \\
\hline No growth & 45 & 90 \\
\hline Total & $\mathbf{5 0}$ & $\mathbf{1 0 0}$ \\
\hline \multicolumn{2}{|c|}{ Table 3: Bacteriology upon Inoculation } \\
into Sterile Container
\end{tabular}

\begin{tabular}{|c|c|c|}
\hline Organisms & Frequency & Percentage \\
\hline E. coli & 1 & 2 \\
\hline E. coli + Pseudomonas & 1 & 2 \\
\hline Pseudomonas & 4 & 8 \\
\hline No growth & 44 & 88 \\
\hline Total & 50 & 100 \\
\hline \multicolumn{3}{|c|}{$\begin{array}{c}\text { Table 4: Bacteriology in Blood Culture Broth (Enrichment } \\
\text { Media) }\end{array}$} \\
\hline
\end{tabular}

\begin{tabular}{|c|c|c|}
\hline Organisms & Frequency & Percentage \\
\hline $\begin{array}{c}\text { Non-Fermenter Gram Negative } \\
\text { Bacilli }\end{array}$ & 2 & 4 \\
\hline No Growth & 48 & 96 \\
\hline Total & 50 & 100 \\
\hline Table 5: Bacteriology in RCM (Enrichment Media) \\
\hline
\end{tabular}

There was one subject in whom the Routine Culture Media could not detect a growth, which was detected upon inoculation in Blood Culture Media. The organism identified here was Pseudomonas, which was not picked up by the inoculation in sterile container.

There were two organisms which were detected upon inoculation into the RCM media and these were not detected by the routine inoculation in sterile container. These were Non-Fermenter Gram Negative bacilli.

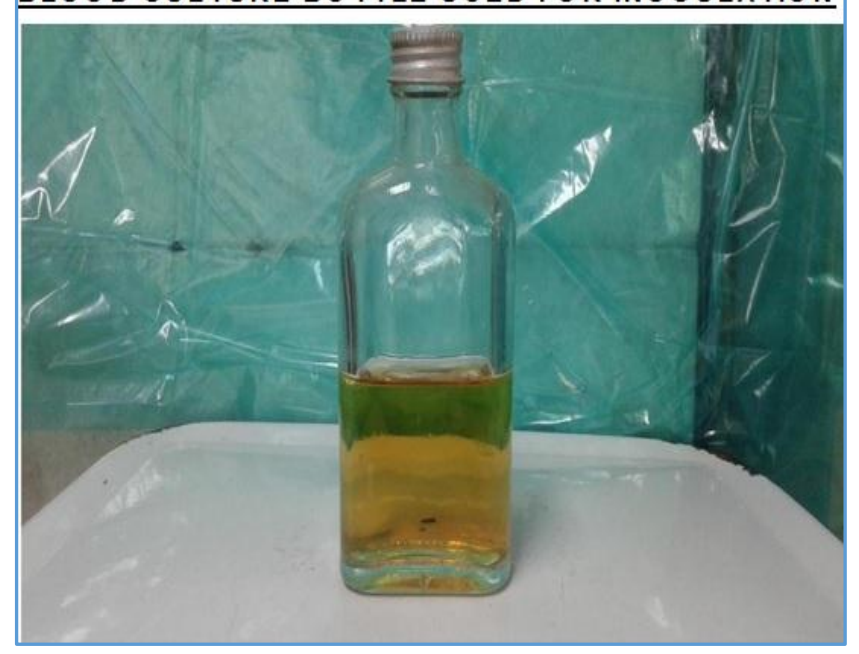



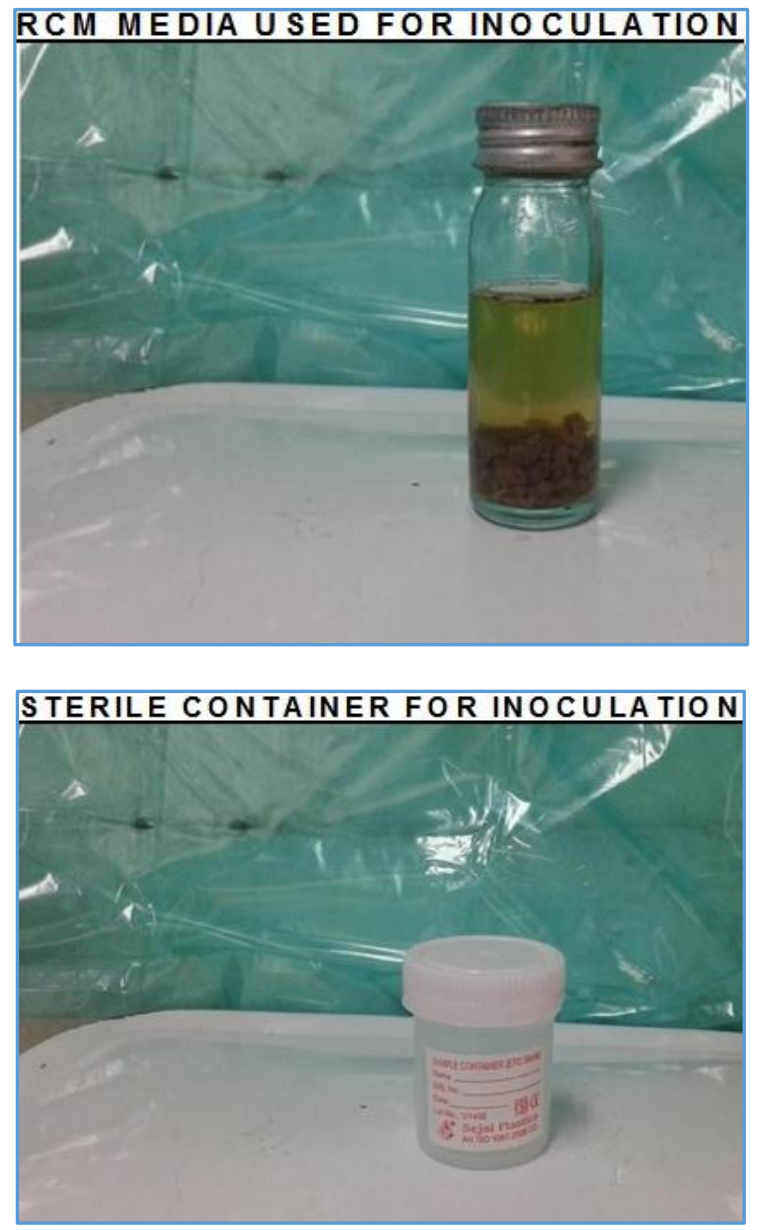

\section{DISCUSSION}

Of the 50 subjects in our study, there were 36 males (72\%) and 14 females (28\%). The maximum number of patients were concentrated in the age group of 20 to 30 years $(n=19$, $38 \%)$.

Zablockis $\mathrm{R}^{(9)}$ et al studied 120 patients with pleural empyema $(n=67)$ and complicated parapneumonic pleural effusion $(n=53)$. Most patients with pleural empyema and complicated parapneumonic pleural effusion were middle aged (53+/-17 years); men were twice as likely as women to have these conditions.

Out of the 50 samples inoculated into the sterile specimen container in our study, there was a growth obtained in 5 samples. This amounted to $10 \%$ yield. In one sample, it was E. coli (2\%). A combined growth of E. coli and Pseudomonas in the other $(2 \%)$, and Pseudomonas was isolated in three samples (6\%). Thus all the organisms isolated were Gram Negative. Pseudomonas was the most common isolated organism.

An Indian study(10) of 2906 pleural fluid samples was done, of which 459 (16\%) samples were culture positive. The number of Gram-negative and positive organisms isolated was $412(86.4 \%)$ and 65 (13.6\%), respectively. The Gramnegative organisms were Acinetobacter spp. (27.7\%), Pseudomonas aeruginosa (23.9\%) and Klebsiella spp. (12.6\%). Staphylococcus aureus (9.6\%) was the most frequent Gram-positive organism.

Out of the 50 samples inoculated into the blood culture media in our study, there was a growth obtained in 6 samples. This amounted to $12 \%$ yield. In one sample, it was E. coli (2\%). A combined growth of E. coli and Pseudomonas in the other (2\%) Pseudomonas was isolated in four samples $(8 \%)$. There was one sample in which an additional organism was not picked up by inoculation into the routine sterile container, was picked up by inoculation in the blood culture media.

In another study(11) the bacterial isolation rates on inoculating in standard culture was $38 \%$, i.e. 20 positive out of 53 . While it increased to $58 \%$, i.e. 31 out of 53 cases on inoculation into the blood culture bottle, thus there was a $20.8 \%$ increase in the yield $(\mathrm{p}<0.001)$ on comparison with the standard laboratory culture. Blood culture bottle inoculation also added microbiological value to standard culture in a further two (3.8\%) cases by identifying additional microbiologically relevant organisms when the standard culture was already positive (one Enterococcus spp. and one Streptococcus spp.-when standard culture was solely anaerobic).

In addition to increasing the organism identification rate over standard culture at the recruiting hospital, blood culture identified additional clinically important co-infecting bacteria in $2 / 53(3.8 \%)$ cases. In the first case standard culture isolated Methicillin-Resistant aureus (MRSA), Pseudomonas aeruginosa and anaerobes, whereas bottle culture identified an Enterococcus spp.- which was of clinical value given the resistance patterns these organisms exhibit. In the second standard culture grew only anaerobes and bottle culture identified an aerobic growth of an organism from the Streptococcus anginosus group.

Xiol $\mathrm{X}^{(12)}$ et al reviewed pleural fluid culture of cirrhotic patients with pleural effusion by two methods: conventional and modified (inoculation of pleural fluid into a blood culture bottle at the bedside); 16 of the 120 (13 percent) patients admitted with hydrothorax had 24 episodes of spontaneous bacterial empyema. Pleural fluid culture was positive by the conventional method in 8 episodes ( 33 percent) and by the modified method (blood culture inoculation) in 18 (75 percent) $(\mathrm{P}=.004)$. Thus it was found that culture of pleural fluid by inoculating into a blood culture bottle at the bedside had a higher yield.

Ferrer $\mathrm{A}^{(13)}$ et al evaluated the aetiology of pleural effusions and two different methods of pleural fluid transport and culture. There were a total of 245 samples inoculated into a transport vial, an aerobic and anaerobic blood culture vial and finally into a sterile tube. Of the total, $38(15.5 \%)$ samples were positive for microorganisms. Of the 25 positive samples excluding those samples that grew mycobacteria, nine (36\%) were positive exclusively in the blood culture vials. Twelve organisms were isolated, only one of which did not grow in the anaerobic vial. Two $(8 \%)$ samples were positive by conventional culture only and 14 (56\%) were positive by both methods. The microorganism isolation rate obtained with use of blood culture vials was greater than that obtained with the conventional method of transport and culture. In conclusion, in this study for microbiological study of pleural fluid it seems appropriate to inoculate all samples including non-purulent samples into both a sterile tube and an anaerobic blood culture vial.

Out of the 50 samples inoculated into the RCM media in our study, there was a growth obtained in 2 samples. That amounts to a $4 \%$ yield. The organisms were Non-Fermenter Gram Negative bacilli. These were not picked up by inoculation into sterile specimen container. 
Brook I(14) studied the microbiology and clinical features of empyema retrospectively in 197 patients whose specimens yielded bacterial growth after inoculation for aerobic and anaerobic bacteria. Aerobic bacteria were isolated in 127 (64\%), anaerobic in 25 (13\%) and mixed growth in 45 (23\%). These data highlight the importance of anaerobic bacteria in selected cases of empyema.

The total yield of this study was $16 \%$ (i.e. 8 samples showed a growth out of 50$)$. Of these 5 samples (10\%) had detected a growth that was common to inoculation into Routine Sterile container and Blood Culture Media. One sample (2\%) had shown a growth only upon inoculation into the blood culture media. Two samples (4\%) had shown a growth only upon inoculation into RCM media.

In our study, the 42 samples (84\%) did not show any growth at all upon inoculation into either the sterile container or the enrichment media. A wide variation in negative cultures has been observed worldwide ranging from

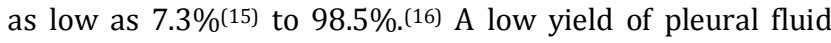
cultures is in concurrence with various studies by J Sonali et al.(17)

\section{CONCLUSIONS}

Pleural fluid cultures are commonly ordered on pleural fluid samples, but the frequency of true-positive results is very low. Without a -gold standard with which one can compare these results, it is difficult to calculate the sensitivity of the cultures.

Recommendations regarding the use of pleural fluid cultures are not well established and are somewhat contradictory. Sahn(18) recommended that - Gram, KOH [potassium hydroxide] and AFB stain and pleural fluid cultures should be done only when an infection is suspected with an exudative effusion.

However, a statement from the British Thoracic Society recommended that Gram stain, AFB stain and microbiologic culture be obtained on all pleural fluid samples.(19)

There was a two percent (2\%) increase in the yield by inoculation into blood culture broth (enrichment media). Upon inoculation into the Robertson's Cooked Meat Media, there were two organisms which were isolated which were Non-Fermenter Gram negative bacilli. Thus, it amounted to $4 \%$ cases. These were not detected upon inoculation into sterile specimen container.

All the isolated organisms were Gram Negative organisms in both conventional as well as enrichment media inoculation.

Even though only a $2 \%$ increase in the yield is seen in this study upon inoculation into blood culture broth and $4 \%$ yield in RCM MEDIA (not picked up by routine sterile container inoculation) it becomes very important for clinicians. This will help in diagnosing the microorganisms not detected by conventional inoculation and help in institution of appropriate treatment based on the antibiotic sensitivity pattern, thereby reducing the morbidity and mortality.

A bigger sample size will help to identify additional cases and to determine whether there would be any significant difference upon inoculation into enrichment media.

\section{REFERENCES}

1. Light RW, Girard WM, Jenkinson SG, et al. Parapneumonic effusions. Am J Med 1980;69(4):507-12.
2. Sahn SA. Diagnosis and management of parapneumonic effusions and empyema. Clin Infect Dis 2007;45(11): 1480-6.

3. Ahmed RA, Marrie TJ, Huang JQ. Thoracic empyema in patients with community-acquired pneumonia. Am J Med 2006;119(10):877-83.

4. Tsang KY, Leung WS. Chan VL, et al. Complicated parapneumonic effusion and empyema thoracis: microbiology and predictors of adverse outcomes. Hong Kong Med J 2007;13(3):178-86.

5. Jerng JS, Hsueh PR, Teng LJ, et al. Empyema thoracis and lung abscess caused by viridans streptococci. Am J Respir Crit Care Med 1997;156(5):1508-14.

6. Isenberg HD, Washington JA II, Doern GV, et al. Specimen collection and handling. In: Balows A, Hausler WJ, Herrman K, et al, eds. Manual of clinical microbiology. $5^{\text {th }}$ edn. Washington, D.C: American Society for Microbiology 1991:p. 15-28.

7. von Essen R. Blood culture bottle improves microbe isolation rate from joint fluids. Am J Clin Pathol 1989;91(4):501-3.

8. Yagupsky P, Dagan R, Howard CW, et al. High prevalence of Kingella kingae in joint fluid from children with septic arthritis revealed by the BACTEC blood culture system. J Clin Microbiol 1992;30(5):1278-81.

9. Zablockis R, Petruskeviciene R, Nargela RV. The causes and risk factors of pleural empyema and complicated parapneumonic pleural effusion. Medicina (Kaunas) 2010;46(2):113-9.

10. Mohanty S, Kapil A, Das BK. Bacteriology of parapneumonic pleural effusions in an Indian hospital. Trop Doct 2007;37(4):228-9.

11. Menzies SM, Rahman NM, Wrightson JM, et al. Blood culture bottle culture of pleural fluid in pleural infection. Thorax 2011;66(8):658-62.

12. Xiol X, Castellví JM, Guardiola J, et al. Spontaneous bacterial empyema in cirrhotic patients: a prospective study. Hepatology 1996;23(4):719-23.

13. Ferrer A, Osset J, Alegre J, et al. Prospective clinical and microbiological study of pleural effusions. Eur J Clin Microbiol Infect Dis 1999;18(4):237-41.

14. Brook I, Frazier EH. Aerobic and anaerobic microbiology of empyema. A retrospective review in two military hospitals. Chest 1993;103(5):1502-7.

15. Alfageme I, Muñoz F, Peña N, et al. Empyema of the thorax in adults. Etiology, microbiologic findings, and management. Chest 1993;103(3):839-43.

16. Barnes TW, Olson EJ, Morgenthaler TI, et al. Low yield of microbiologic studies on pleural fluid specimens. Chest 2005;127(3):916-21.

17. Jain S, Banavaliker JN. Empyema thoracis: bacteriological analysis of pleural fluid from the largest chest hospital in Delhi. IOSR Journal of Dental and Medical Sciences 2013;3(6):46-51.

18. Sahn SA. State of the art: the pleura. Am Rev Respir Dis 1988;138(1):184-234.

19. Maskell NA, Butland RJ. Pleural Diseases Group, et al. BTS guidelines for the investigation of a unilateral pleural effusion in adults. Thorax 2003;58(Suppl 2):ii8-ii17. 